\title{
VLA OBSERVATIONS OF DISKS AND JETS IN YOUNG CLOSE BINARIES
}

\author{
G. Anglada ${ }^{1}$
}

RESUMEN

Presentamos resultados observacionales de alta resolución angular de las fuentes SVS 13, NGC 1333 VLA 2 y L723 VLA 2 obtenidos con el VLA. Nuestros resultados indican que estas fuentes son sistemas binarios compactos (con una separación de 25-75 AU) y muy jóvenes, y sugieren que el proceso de desarrollo de discos y chorros puede ocurrir de modo distinto para cada componente del sistema binario.

\section{ABSTRACT}

We show high-angular resolution results from VLA observations of the sources SVS 13, NGC 1333 VLA 2, and L723 VLA 2. Our results suggest that these sources are very young close (25-75 AU separation) binary systems and that the development of disks and jets can proceed in a different way for each component of the binary system.

\section{Key Words: BINARIES: CLOSE - ISM: JETS AND OUTFLOWS - RADIO CONTINUUM: ISM - STARS: FORMATION}

\section{INTRODUCTION}

Collimated outflows, which are ubiquitously found in star-forming regions, appear to be a necessary stage in the early evolution of all the stars. Although jets and outflows can be traced up to huge distances (several pc) from their driving source, the region where the launch and collimation processes take place (10-100 AU from the origin) is still poorly investigated. This region is usually hidden at optical wavelengths due to very high extinction, particularly for the youngest objets, making its observation difficult. Very Large Array (VLA) observations, at wavelengths of $7 \mathrm{~mm}$ and longer, provide a way to investigate this launching region. Observation of the continuum emission at wavelengths longer than $1 \mathrm{~cm}$ usually traces the emission from collimated, ionized jets, while the emission from the disk of dust can be traced by the observations at $7 \mathrm{~mm}$ (see, e.g., Anglada 1996, Rodríguez 1997). In addition, observation of the strong maser emission at $1.3 \mathrm{~cm}$ is very useful to trace the kinematics of both disks and jets (e.g., Torrelles et al. 1998), although it is highly variable and requires very special physical conditions for excitation, being more difficult to interpret.

In this paper we will show some results obtained from VLA observations of close binary systems.

\section{NGC 1333}

The reflection nebula NGC 1333 is associated with a very active star-forming region in the Perseus molecular cloud. The region contains a cluster of

\footnotetext{
${ }^{1}$ Instituto de Astrofísica de Andalucía, CSIC, Spain.
}

about 150 low- to intermediate-mass young stellar objects (YSOs), and about 20 groups of Herbig-Haro systems (see Bally, Devine, \& Reipurth 1996 and references therein). Rodríguez, Anglada, \& Curiel (1999), using the VLA of $\mathrm{NRAO}^{2}$, detected 44 radio continuum sources, most of them associated with objects of the star-forming region (see Fig. 1a).

$$
\text { 2.1. } \operatorname{SVS} 13 \text { (=VLA 4) }
$$

The Herbig-Haro 7-11 system (Herbig 1974; Strom, Grasdalen, \& Strom 1974) is the most studied HH group in the region. The SVS 13 star, originally discovered as a $2.2 \mu \mathrm{m}$ source (Strom, Vrba, \& Strom 1976), is roughly aligned with the chain of HH objects 7-11, and it was assumed to be the exciting source of this classical HH system. SVS 13 has been detected also at optical (e.g., Goodrich 1986; Eislöffel et al. 1991) and $\mathrm{cm}$ wavelengths (source VLA4 in Rodríguez, Anglada, \& Curiel 1997, 1999; see Fig. 1b). However, despite being optically visible, suggesting that it is a relatively evolved young object, SVS 13 is a strong mm source (e.g., Looney, Mundy, \& Welch 2000), and presents other characteristics, such as the presence of an extremely high velocity $\mathrm{CO}$ outflow, that suggest it is a very young stellar object (a Class 0/I source; Bachiller et al. 2000).

Sensitive, subarsecond angular resolution observations at $\mathrm{cm}$ wavelengths reveal that the source is a binary (Anglada, Rodríguez, \& Torrelles 2000), with

\footnotetext{
${ }^{2}$ The National Radio Astronomy Observatory is a facility of the National Science Foundation operated under cooperative agreement by Associated Universities, Inc.
} 


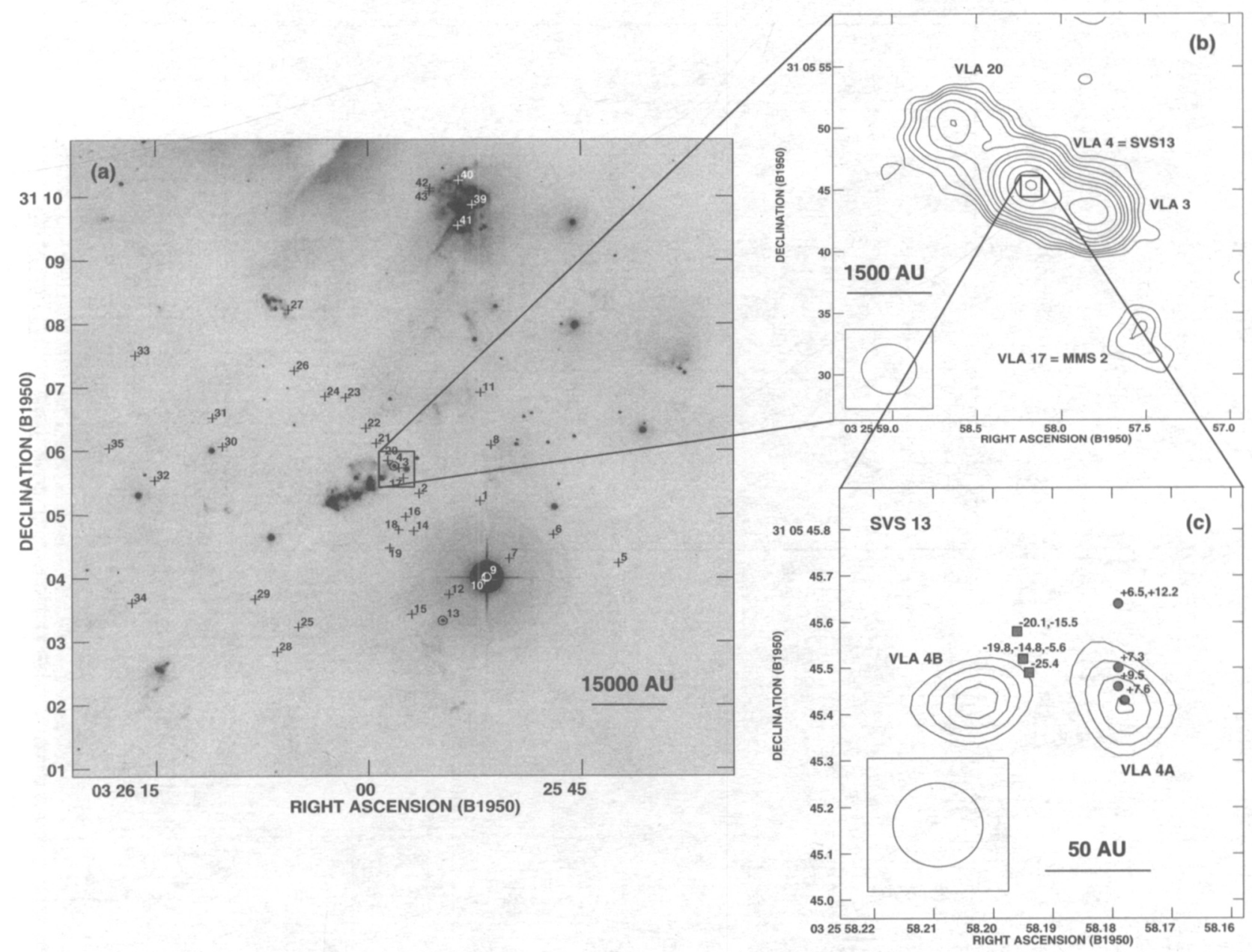

Fig. 1. (a) [SII] image of the region around HH 7-11, obtained by Bally et al.(1996), with the positions of the $\mathrm{cm}$ radio continuum sources detected by Rodríguez et al. (1999) indicated. (b) VLA map at $3.6 \mathrm{~cm}$ of the region near SVS 13 (= VLA 4) obtained by Rodríguez et al. (1999) with an angular resolution of $\sim 5^{\prime \prime}$. (c) Map of SVS 13 at $3.6 \mathrm{~cm}$, obtained by Anglada et al. (2000) with an angular resolution of $\sim 0$ ". 2 , showing that the source is a binary with the two components (VLA 4A and VLA 4B) separated by $\sim 65 \mathrm{AU}$. Overlapped are shown the positions and velocities of water maser spots (Rodríguez et al. 2002); note that the spots with $V_{\mathrm{LSR}}$ velocities closer to the velocity of the ambient cloud (small circles) are spatially associated with VLA 4A, while those with blueshifted velocities with respect to the ambient cloud (small squares) are spatially associated with VLA $4 \mathrm{~B}$, suggesting that this last source is the driving source of a maser outflow.

the two components separated by $\sim 65 \mathrm{AU}$ (for an assumed distance of $220 \mathrm{pc}$ ). Furthermore, the western component (VLA 4A) appears to coincide with the visible star, while the eastern component (VLA $4 \mathrm{~B}$ ) is the dominant source at $\mathrm{mm}$ wavelengths (see Anglada et al. 2003, 2004a). This result suggests that in the case of the SVS13 binary source, one of the components is developing a disk of dust, while the other component is not. This is in contrast with the case of the L1551-IRS5 source, where both components of the close binary system are developing protoplanetary disks (Rodríguez et al. 1998). An- other difference is that in the case of the L1551-IRS5 binary, each component energizes a collimated jet (Rodríguez et al. 2003), while in SVS 13 the distribution of water masers suggests that only VLA 4B (the component with the disk) is associated with a maser outflow (see Fig. 1c).

\section{2. $V L A 2$}

The cm source VLA 2 (Rodríguez et al. 1997, 1999), located $\sim 30^{\prime \prime}$ southwest of SVS 13 (see Fig. 1a), is the counterpart of the mm source MMS 3 (Chini et al. 1997). The sensitive VLA map obtained by Reipurth et al. (2002) at $3.6 \mathrm{~cm}$ reveals a faint, 


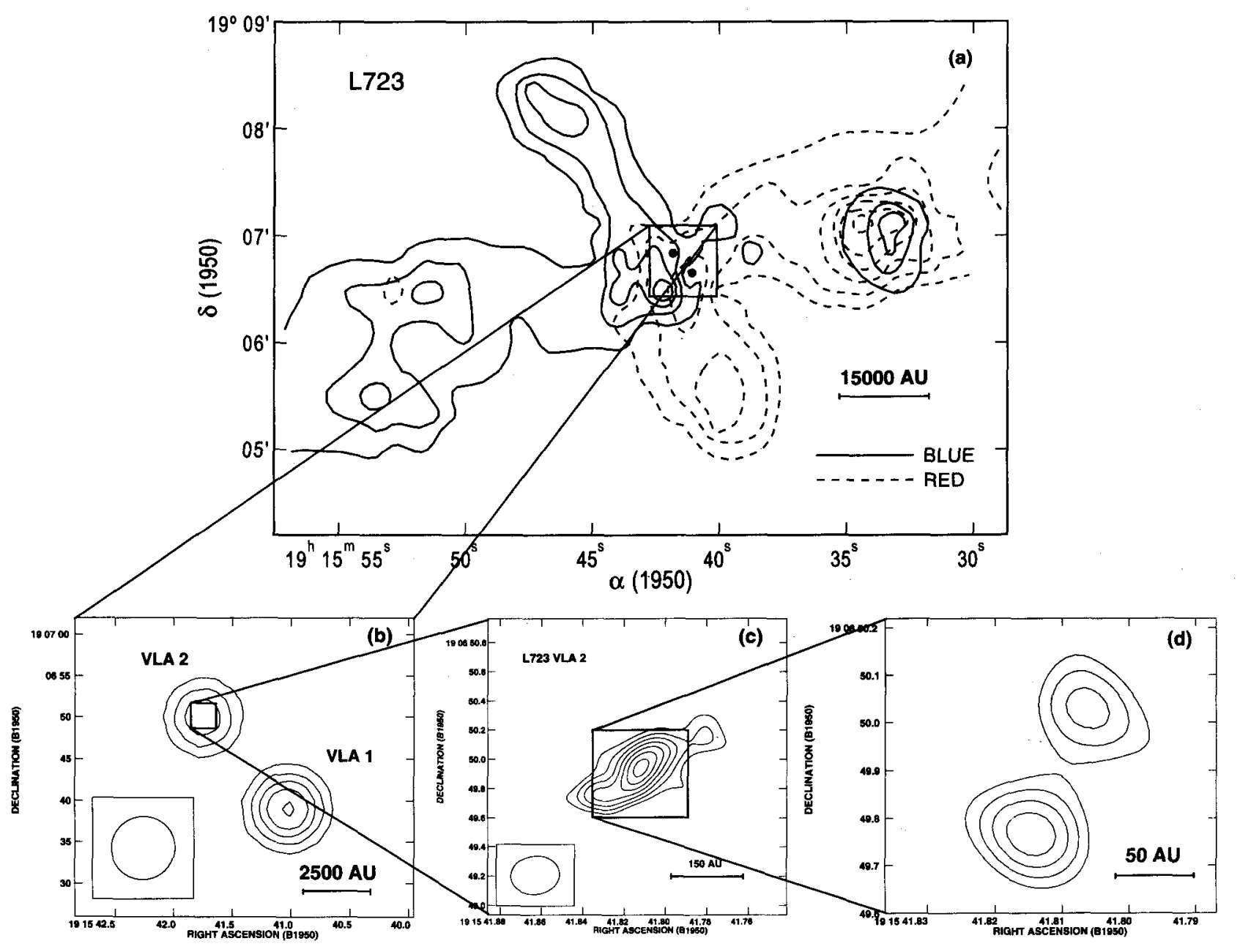

Fig. 2. (a) CO map of the quadrupolar molecular outflow in L723 (adapted from Avery et al. 1990). (b) VLA map at $3.6 \mathrm{~cm}$ of the radio continuum sources towards the center of the outflow, observed by Anglada et al. (1991) with the VLA with an angular resolution of $\sim 8^{\prime \prime}$. $(c)$ A high angular resolution $\left(\sim 0^{\prime \prime} 3\right)$ map of the source VLA 2 at $3.6 \mathrm{~cm}$, revealing that this source is a radio jet elongated in the direction of the main pair of outflow lobes (from Anglada et al. 1996). (d) Close up of the core of the VLA 2 source, obtained through $3.6 \mathrm{~cm}$ VLA observations of improved angular resolution $(0.2)$ and sensitivity (Anglada et al. 2004b), showing two sources separated by $\sim 65$ AU.

bipolar jet with a clear wiggling, extending $\sim 1000$ $\mathrm{AU}$ in the north-south direction (see their Fig. 5). This wiggling is a likely signpost for the presence of a binary system, unresolved by the VLA beam at 3.6 $\mathrm{cm}\left(\sim 00^{\prime \prime} 3\right)$.

An additional indication for the existence of two protostars is provided by the VLA observations of the $1.3 \mathrm{~cm}$ continuum and water maser emission (with an angular resolution of $0 . \prime 08$ ), that reveal two groups of water maser spots separated by $\sim 0$.' 1 $(\sim 25 \mathrm{AU})$ towards the core of the $3.6 \mathrm{~cm}$ source. These two groups of masers may be tracing the positions of the two components of the binary system (see Anglada et al. 2003, 2004a for further details).

\section{L723}

The molecular outflow in L723 is a prototypical example of a quadrupolar outflow, as can be seen in the CO map of Avery, Hayashi \& White (1990). The outflow presents two pairs of CO lobes: a larger pair aligned roughly in the NW-SE direction, and a smaller one, aligned roughly in the NESW direction (see Fig. 2a). The presence of two pairs of bipolar lobes suggested that a binary system could be powering this outflow. VLA observations with relatively low angular resolution $\left(\sim 8^{\prime \prime}\right)$ revealed two radio sources (VLA 1 and VLA 2), separated by $\sim 15^{\prime \prime}(4500 \mathrm{AU}$ at $300 \mathrm{pc})$, towards the center of the outflow (see Fig. 2b). Further 
VLA observations with much higher angular resolution (Anglada, Rodríguez, \& Torrelles 1996) revealed that the source VLA 2 is elongated along the NW$\mathrm{SE}$ direction, suggesting that it is a thermal radio jet responsible for the excitation of only the NWSE pair of CO lobes. However, source VLA 1 appeared unresolved with the VLA angular resolution of $\sim 0$ " 3 , with independent evidence indicating that this source is not directly related to the outflow excitation (e.g., Girart et al. 1997; Lee et al. 2002). Additional VLA observations with improved angular resolution suggest that the core of the VLA 2 source splits into two components separated by $\sim 75$ AU (Anglada et al. 2004b; see Fig. 3d). A long-term monitoring suggests that one of these components is responsible for the excitation of the larger pair of CO lobes, while the other component appears to be driving the smaller one.

Support from MCYT grant AYA2002-00376, and from Junta de Andalucía (Spain) is acknowledged.

\section{REFERENCES}

Anglada, G. 1996, in ASP Conf. Ser. 93, Radio Emission from the Stars and the Sun, ed. A. R. Taylor \& J. M. Paredes (San Francisco: ASP), 3

Anglada, G., Estalella, R., Rodríguez, L. F., Torrelles, J. M., López, R., \& Cantó, J. 1991, ApJ, 376, 615

Anglada, G., Rodríguez, L. F., \& Torrelles, J. M. 1996, ApJ, 473, L123

Anglada, G., Rodríguez, L. F., \& Torrelles, J. M. 2000, ApJ, 542, L123

Anglada, G., Rodríguez, L. F., Torrelles, J. M., Estalella, R., Beltrán, M. T., \& Ho, P. T. P. 2003, RevMexAA(SC), 15, 97

Anglada, G., Rodríguez, L. F., Osorio, M., Torrelles, J. M., Estalella, R., Beltrán, M. T., \& Ho, P. T. P. 2004a, ApJ (Letters), submitted
Anglada, G. et al. 2004b, in preparation

Avery, L. W., Hayashi, S. S., \& White, G. J. 1990, ApJ, 357,524

Bally, J., Devine, D., \& Reipurth, B. 1996, ApJ, 473, L49

Bachiller, R., Gueth, F., Guilloteau, S., Tafalla, M., \& Dutrey, A. 2000, A\&A, 362, L33

Chini, R., Reipurth, B., Sievers, A., Ward-Thompson, D., Haslam, C. G. T., Kreysa, E., \& Lemke, R. 1997, A\&A, 325, 542

Eislöffel, J., Günther, E., Hessman, F. V., Mundt, R., Poetzel, R., Carr, J. S., Beckwith, S., \& Ray, T. P. 1991, ApJ, 383, L19

Girart, J. M., Estalella, R., Anglada, G., Torrelles, J. M., Ho, P. T. P., \& Rodríguez, L. F. 1997, ApJ, 489, 73

Goodrich, R. W. 1986, AJ, 92, 885

Herbig, G. H. 1974, Lick Observatory Bulletin, 658, 1

Lee, C., Mundy, L. G., Stone, J. M., \& Ostriker, E. C. 2002, ApJ, 576, 294

Looney, L. W., Mundy, L. G., \& Welch, W. J. 2000, ApJ, 529,477

Reipurth, B., Rodríguez, L. F., Anglada, G., \& Bally, J. 2002, AJ, 124, 1045

Rodríguez, L. F. 1997, in IAU Symp. 182: Herbig-Haro Flows and the Birth of Stars, ed. B. Reipurth \& C. Bertout (Dordrecht: Kluwer), 83

Rodríguez, L. F., Anglada, G., \& Curiel, S. 1997, ApJ, 480, L125

Rodríguez, L. F., Anglada, G., \& Curiel, S. 1999, ApJS, 125,427

Rodríguez, L. F., Anglada, G., Torrelles, J. M., MendozaTorres, J. E., Haschick, A. D., \& Ho, P. T. P. 2002, A\&A, 389, 572

Rodríguez, L. F. et al. 1998, Nature, 395, 355

Rodríguez, L. F., Porras, A., Claussen, M. J., Curiel, S., Wilner, D. J., \& Ho, P. T. P. 2003, ApJ, 586, L137

Strom, S. E., Grasdalen, G. L., \& Strom, K. M. 1974, ApJ, 191, 111

Strom, S. E., Vrba, F. J., \& Strom, K. M. 1976, AJ, 81, 314

Torrelles, J. M., Gómez, J. F., Rodríguez, L. F., Curiel, S., Anglada, G., \& Ho, P. T. P. 1998, ApJ, 505, 756

\section{DISCUSSION}

Hanawa - Can you tell me whether S1/S13 VLA 4B, the component having the dust disk, is the primary or the secondary?

Anglada - On the basis of the current radio continuum data it is not possible to distinguish the primary from the secondary. However, with future observations it could be possible to measure proper motions and to determine the absolute orbital motion (as has been done already in a few sources, as explained in the previous talk by Luis Rodríguez), allowing to identify the secondary. From a different approach, the theoretical results of $\mathrm{M}$. Bate would favor VLA $4 \mathrm{~B}$ (the component having the disk) as the primary, if one assumes a low value for the specific angular momentum of the system.

Guillem Anglada: Instituto de Astrofísica de Andalucía, CSIC, Camino Bajo de Huétor 24, E-18008 Granada, Spain (guillem@iaa.es). 